\title{
Technology Automation of Satellite Data Processing for Operational Mapping of Water and Ice Surfaces
}

\author{
Anastasiya Voronova, Sergey Kuzminykh \\ Siberian Center of SRC "Planeta", Novosibirsk, Russia \\ 35voran@gmail.com
}

\begin{abstract}
The process of technology automation of satellite data thematic processing presented in the article includes two main stages: the development of classification algorithm and the creation of a software module based on them. At the first stage, algorithm (decision tree) was developed to isolate water surfaces in conditions of destroyed ice cover using medium resolution satellite images (Landsat-8, OLI). The article presents the final dendrogram, examples of the thematic maps and the results of accuracy assessment in comparison with standard masks and supervised classification.

At the second stage, a software module based on the IDL language was created. This module allows to perform the following tasks automatically: primary data processing, classification, "cleaning" the results, exporting to a vector format.
\end{abstract}

Keywords: decision tree, classification, indices, ice, NDVI, NDII, OLI, Landsat-8

Copyright (C2019 by the paper's authors. Copying permitted for private and academic purposes 


\title{
АВТОМАТИЗАЦИЯ ТЕХНОЛОГИИ ОБРАБОТКИ СПУТНИКОВЫХ ДАННЫХ ДЛЯ ОПЕРАТИВНОГО КАРТОГРАФИРОВАНИЯ ВОДНОЙ И ЛЕДОВОЙ ПОВЕРХНОСТЕЙ
}

\author{
А.Е. Воронова, С.А. Кузьминьхх \\ Сибирский центр НИЦ «Планета», Новосибирск, Россия \\ 35voran@gmail.com
}

Представленный в статье процесс автоматизации технологии тематической обработки спутниковых данных включает в себя разработку порогового алгоритма классификации и создание на его основе программного модуля.

На первом этапе был разработан пороговый алгоритм (дерево решений) для выделения водных поверхностей в условиях разрушенного ледового покрова по спутниковым изображениям среднего пространственного разрешения (Landsat-8, OLI, TIRS). В статье приведена итоговая дендрограмма алгоритма, полученные тематические карты и результаты оценки точности разработанного алгоритма в сравнении со стандартными масками и обучаемой классификацией.

На втором этапе был создан программный модуль на базе языка IDL. Данный модуль позволяет выполнять в автоматическом режиме следующие задачи: первичную обработку данных, классификацию с помощью выбранного дерева решений, «очистку» результатов классификации, экспорт целевого класса в векторный формат.

Ключевые слова: дерево решений, классификация, индексы, лед, NDVI, NDII, OLI, Landsat-8

\section{Введение}

Оперативный спутниковый мониторинг состояния водных объектов во время весенних ледовых явлений играет важную роль в предупреждении и ликвидации чрезвычайных ситуаций. При этом время, затраченное на подготовку тематических карт, становится ключевым фактором обеспечения ежедневного мониторинга. В общем виде технология подготовки тематической продукции включает в себя три основных этапа: подготовка данных, классификация, экспорт данных. В зависимости от исходных данных, эти этапы могут включать в себя различные операции. Самым времязатратным этапом является процесс классификации изображения с целью выделения классов «воды», «льда» и «облачности».

В мировой практике широкое применение для создания масок воды, снега и облачности получили пороговые алгоритмы. Таким образом, например, Европейское космическое агентство (ESA) обеспечивает обработку изображений со спутника Sentinel-2 до уровня «2А». В таких алгоритмах зачастую используются различные спектральные индексы для задания пороговых значений. В настоящий момент существует ряд работ, посвященных применению спектральных индексов для идентификации водной поверхности, как у отечественных авторов [1], так и у зарубежных [2, 3]. Однако в данных статьях рассматриваются водные объекты, не покрытые льдом, и поэтому вопрос, насколько точно по данным индексам можно отделить ледовую поверхность от водной, остается открытым. Кроме того, как показывает практика, многие алгоритмы выдают различные результаты в зависимости от геоклиматических условий исследуемого региона.

Целью данной работы является автоматизация технологии тематической обработки спутниковых изображений. Основными задачами являются разработка порогового алгоритма 
идентификации целевых классов («вода», «лед», «снег») в условиях геоклиматических особенностей сибирского региона и создание программного модуля для автоматизации выполнения классификации и других рутинных процедур (первичная обработка, экспорт данных в векторный формат).

\section{Исследуемый регион}

Сибирский центр ФГБУ «НИЦ «Планета» обеспечивает оперативный мониторинг обширных территорий Уральского и Сибирского федеральных округов. Наиболее пристального внимания во время весенних ледовых явлений требуют пойменные территории реки Оби и ее притоков. Вскрытие реки происходит в период со второй половины марта до начала июня и совпадает с волной половодья. Весенний ледоход часто сопровождается заторами и выходами воды на пойму. При максимальных уровнях половодья разливы на пойме в нижнем и среднем течении Оби достигают нескольких десятков километров, что может приводить к затоплению населенных пунктов и сельскохозяйственных угодий [4].

\section{Данные}

В качестве данных использовались снимки среднего разрешения с космического аппарата Landsat-8 (OLI, TIRS). Характеристики каналов радиометров OLI и TIRS приведены в таблице 1 [5].

Таблица 1. Характеристики каналов OLI и TIRS (Landsat-8)

\begin{tabular}{|c|c|c|}
\hline Каналь & Длина волны (мкм) & $\begin{array}{c}\text { Пространственное } \\
\text { разрешение (м) }\end{array}$ \\
\hline Coastal/Aerosol & $0.43-0.45$ & 30 \\
\hline Blue & $0.45-0.51$ & 30 \\
\hline Green & $0.53-0.59$ & 30 \\
\hline Red & $0.64-0.67$ & 30 \\
\hline NIR & $0.85-0.88$ & 30 \\
\hline SWIR1 & $1.57-1.65$ & 30 \\
\hline SWIR2 & $2.11-2.29$ & 30 \\
\hline panchromatic & $0.50-0.68$ & 15 \\
\hline Cirrus & $1.36-1.38$ & 30 \\
\hline Thermal Infrared 1 & $10.60-11.19$ & 100 \\
\hline Thermal Infrared 2 & $11.50-12.51$ & 100 \\
\hline
\end{tabular}

Исходные изображения были преобразованы в ТOA Reflectance стандартными методами обработки программного комплекса ENVI.

\section{Алгоритм}

В ходе исследования стало ясно, что среди специалистов нет единой терминологии относительно спектральных индексов. Некоторые из них имеют одинаковые названия, а некоторые и вовсе «изобретены» дважды. Например, индекс MNDWI, предложенный Hanqiu Xu [6], является, по сути, разработанным Dozier индексом снежного покрова NDSI [7]. При этом в 1996 году было предложено сразу два водных индекса NDWI [8].

В статье [8] также приведены другие примеры одинаковых индексов, основанных на соотношении ближнего (NIR) и коротковолнового (SWIR) инфракрасных каналов, и предложена единая терминология, учитывающая различный диапазон волн ИК-каналов. 
В нашей работе были использованы спектральные индексы NDVI [9] и NDII [8], выбранные с учетом доступных спектральных диапазонов сенсора OLI. Подробное описание индексов приведено в таблице 2.

Таблица 2. Описание спектральных индексов

\begin{tabular}{|c|c|c|c|}
\hline Название индекса & Формула & $\begin{array}{c}\text { Спектральные } \\
\text { диапазоны (мкм) }\end{array}$ & Автор \\
\hline $\begin{array}{c}\text { Normalized Difference } \\
\text { Vegetation Index (NDVI) }\end{array}$ & $\frac{\rho_{\text {NIR }}-\rho_{\text {Red }}}{\rho_{\text {NIR }}+\rho_{\text {Red }}}$ & $\begin{array}{c}\rho_{\text {Red }}: 0,60-0,68 \\
\rho_{\text {NIR }}: 0,85-0,88\end{array}$ & Tucker C.J. (1979) \\
\hline $\begin{array}{c}\text { Normalized Difference } \\
\text { Infrared Index (NDII) }\end{array}$ & $\frac{\rho_{N I R}-\rho_{S W I R}}{\rho_{N I R}+\rho_{S W I R}}$ & $\begin{array}{c}\rho_{\text {NIR }}: 0,85-0,88 \\
\rho_{\text {SWIR }}: 1,55-1,75\end{array}$ & $\begin{array}{c}\text { Kimes et al. (1981) } \\
\text { Hardisky et al. (1983) }\end{array}$ \\
\hline
\end{tabular}

Для проведения исследования были выбраны три сцены Landsat-8 на территорию Томской области в период с мая по июнь 2018 года. Данные сцены характеризуются наличием всех интересующих классов: вода, лед, снег, облачность. Так же стоит отметить, что пойма Оби на данном участке имеет довольно сложный характер подстилающей поверхности, что приводит к ложным результатам применения стандартных масок для Landsat-8 (BQA) и необучаемых классификаций.

В ходе эмпирического исследования был построен пороговый алгоритм (рисунок 1) для выделения классов облачности (clouds), воды (water), льда (ice), снега (snow). Неклассифицированные пиксели обозначаются классом «back». Класс «снег» имеет условное название, и к нему также могут относиться участки светлого льда. Правые ответвления узлов дендрограммы означают выполнение условия, левые - невыполнение. Переменные с литерой «b» соответствуют номерам каналов сенсора OLI, с литерой «t»- сенсора TIRS. Индексы NDVI и NDII рассчитываются согласно формулам в таблице 2.

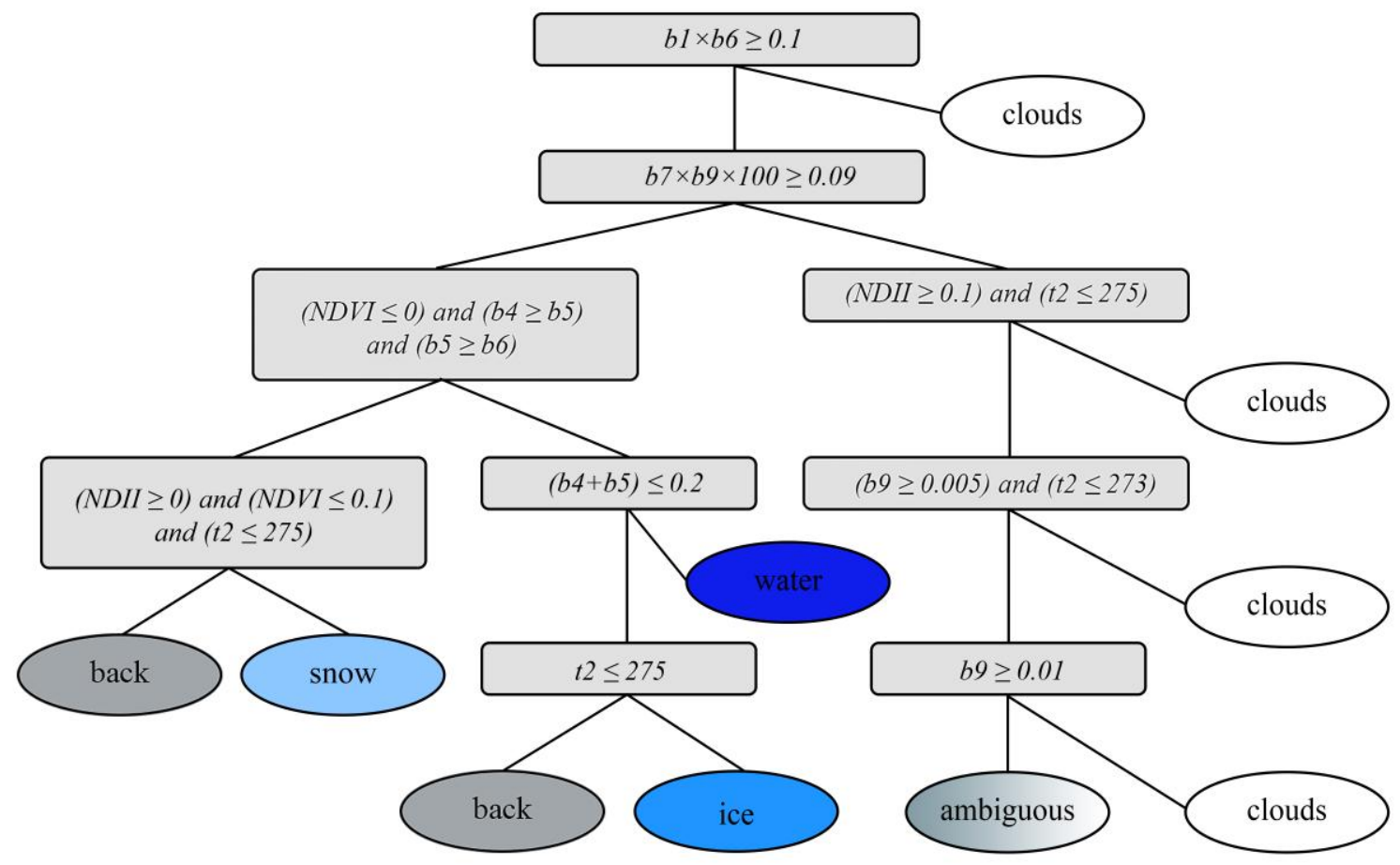

Puс. 1. Пороговый алгоритм для выделения классов облачности, воды, льда, снега по данныlм Landsat-8 (OLI, TIRS)

Помимо целевых классов в алгоритме также присутствует класс «спорных пикселей» (ambiguous), который может быть определен либо как «облачность», либо как «земля» в зависимости от температуры и рельефа подстилающей поверхности. Данный класс занимает 
незначительную долю среди классифицированных пикселей, а окончательное решение о его принадлежности принимает оператор.

\section{Результаты}

На рисунке 2 представлены фрагмент сцены Landsat-8 на территорию Томской области в псевдоцветах (05.05.2018) и результат классификации, наложенный на карту.

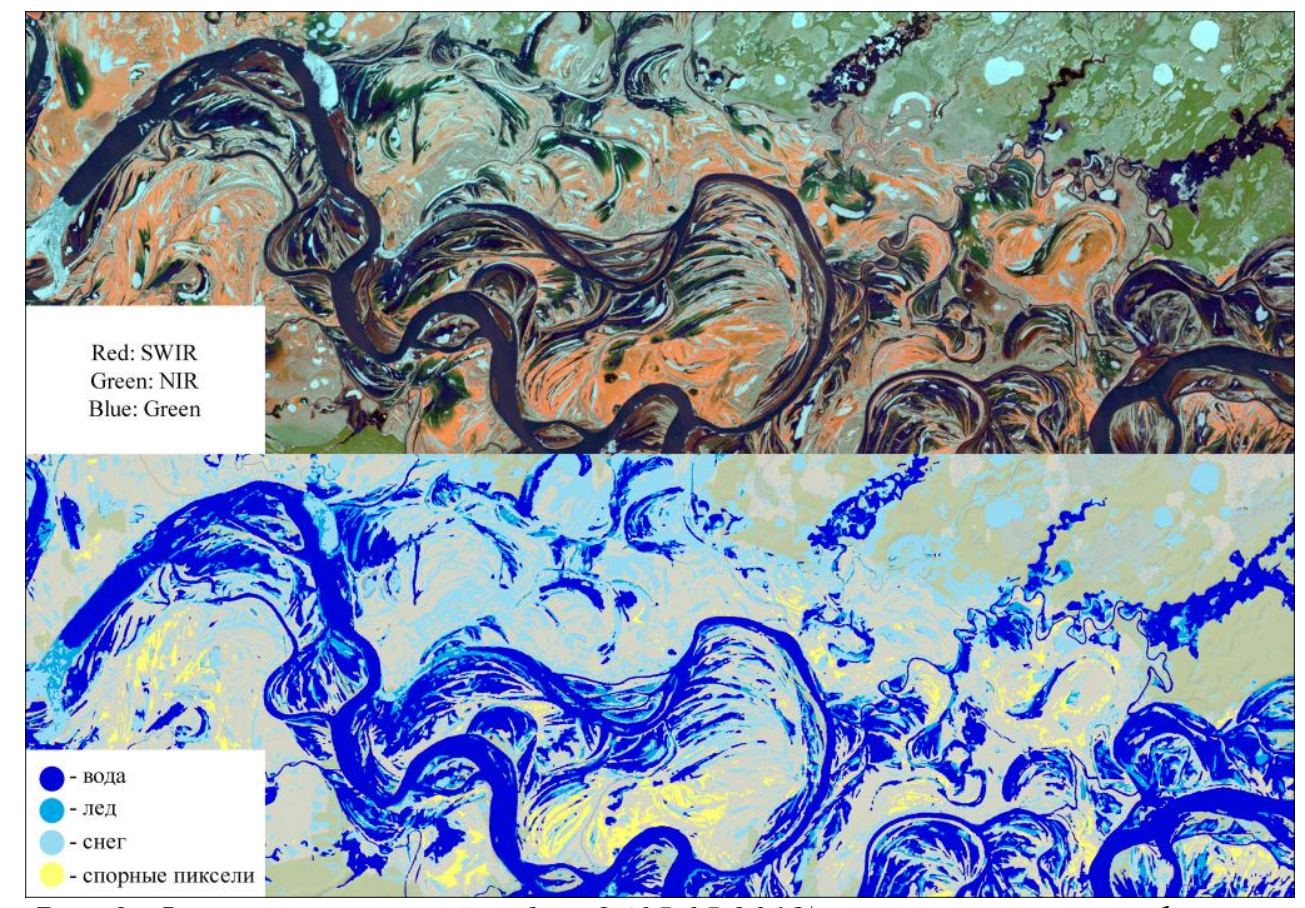

Puc. 2. Фрагмент снимка Landsat-8 (05.05.2018) и результат классификации

Хорошо видно, что в класс «спорных пикселей» попали участки поймы, поэтому при дальнейшей работе над тематической картой указанный класс будет исключен. На рисунке 3 приведены результаты классификации снимка за 08.06.2018.

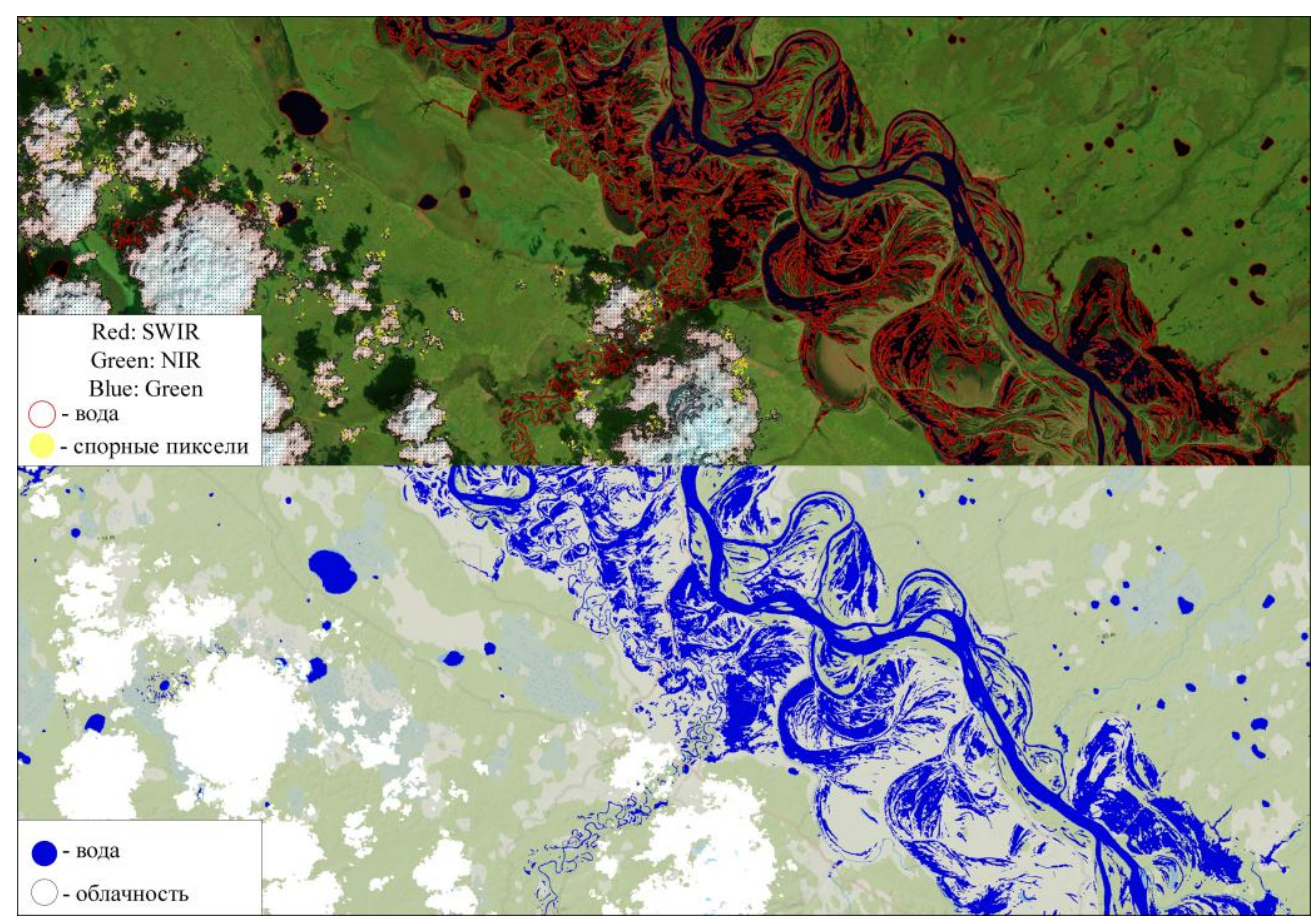

Pис. 3. Фрагмент снимка Landsat-8 (08.06.2018) и результат классификации 
В данном случае к классу «спорных пикселей» относится небольшая часть облачности, при последующей обработке эти пиксели будут присоединены к классу облаков.

\section{Оценка точности}

Для оценки точности классификации был выбран снимок другой территории (озеро Байкал) за 18 мая 2018 года. Из-за отсутствия наземных данных и принципиальной невозможности их получения, за фактические данные были приняты 1118 точек, выбранных опытным дешифровщиком и отнесенных к четырем классам: «облака», «вода», «лед/снег», «прочее». Для сравнительного анализа была проведена оценка точности результатов обучаемой классификации по методу спектрального угла и использования стандартных масок Landsat-8 (BQA). Ввиду отсутствия класса «воды» в BQA, оценка точности для него проводилась по трем классам с использованием 851 точки. На рисунке 4 представлены результаты классификаций и фрагмент снимка с набранными точками.

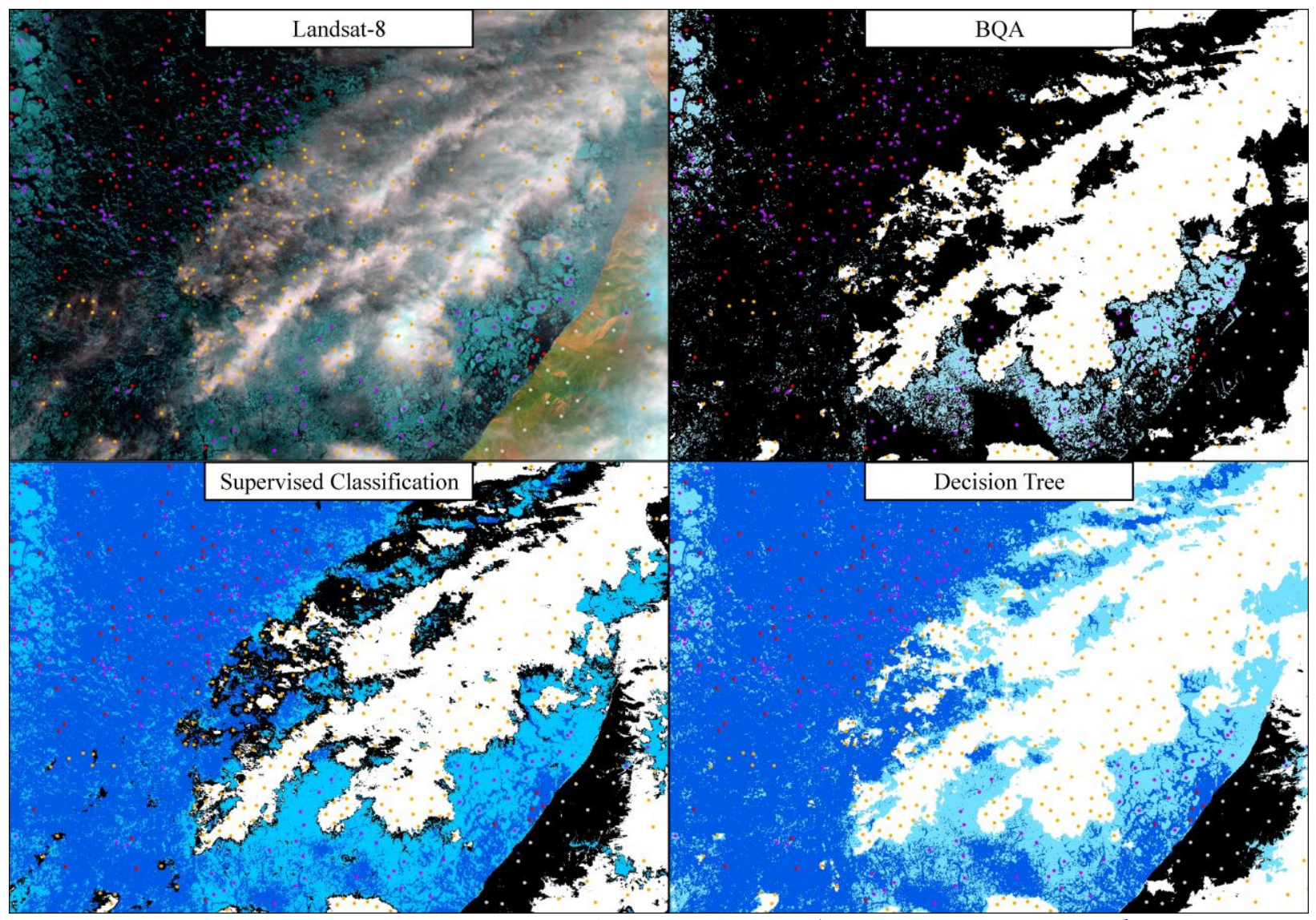

Pис. 4. Фрагмент снимка Landsat-8 (18.05.2018) и результаты классификации

В таблице 3 приведены результаты оценки точности.

Таблица 3. Результаты оценки точности

\begin{tabular}{|c|c|c|c|}
\hline Показатель & $\begin{array}{c}\text { Стандартные } \\
\text { маски }\end{array}$ & $\begin{array}{c}\text { Обучаемая } \\
\text { классификация }\end{array}$ & Дерево решений \\
\hline Количество классов & 3 & 4 & 4 \\
\hline Количество точек & 851 & 1118 & 1118 \\
\hline Общая точность (ТА) & 0,76 & 0,81 & 0,86 \\
\hline Коэффициент Каппа (К) & 0,65 & 0,75 & 0,81 \\
\hline
\end{tabular}


Результаты классификации с помощью разработанного порогового алгоритма (дерева решений) показали наилучшую общую точность $(\mathrm{TA}=0,86)$ и степень согласованности $(\mathrm{K}=0,81)$. Обучаемая классификация лишь в малой степени уступает по точности, однако значительно проигрывает по временным затратам (приблизительно в 5 раз). Применение стандартных масок показало наихудшие результаты: ТА=0,76; K=0,65.

\section{Программный модуль}

На втором этапе автоматизации тематической обработки спутниковых данных был создан программный модуль на базе языка IDL. B него интегрирован представленный алгоритм, а также другие специализированные деревья решений, разработанные в Сибирском центре. Алгоритм работы модуля включает в себя выполнение следующих задач:

- определение исходных данных (тип сенсора);

- первичная обработка данных (радиометрическая калибровка);

- классификация с помощью выбранного дерева решений;

- «очистка» результатов классификации;

- экспорт целевых классов в векторный формат.

В процессе опытной эксплуатации модуля были получены средние значения времени, затрачиваемого на разные этапы обработки. Данные значения сравнивались с временными затратами при работе по традиционной схеме в ручном режиме (обучаемая классификация). Полученные результаты и разница между ними (дельта в процентах) указаны таблице 4.

Таблица 4. Время, затрачиваемое на различные этапы обработки (в минутах)

\begin{tabular}{|c|c|c|c|}
\hline & $\begin{array}{c}\text { Традиционная } \\
\text { схема }\end{array}$ & $\begin{array}{c}\text { Автоматическая } \\
\text { обработка }\end{array}$ & Дельта \\
\hline Первичная подготовка & 4 & 1 & $75 \%$ \\
\hline Классификация & 25 & 3 & $88 \%$ \\
\hline Экспорт данных & 2 & 1 & $50 \%$ \\
\hline Суммарно & 31 & 5 & $84 \%$ \\
\hline
\end{tabular}

Таким образом, разработанный модуль позволяет сократить общее время обработки приблизительно на $80 \%$ благодаря трем факторам: автоматизации рутинных действий, выполняемых оператором, автоматическому выполнению классификации и отсутствию необходимости загрузки (визуализации) изображений, что обычно занимает значительное время.

\section{Заключение}

Разработанная технология позволила значительно сократить время подготовки тематических карт паводковой и ледовой обстановки в оперативном режиме, не жертвуя при этом точностью классификации. Тем не менее, представленный алгоритм требует дальнейшей апробации на более масштабных наборах данных и, возможно, доработки пороговых условий с целью повышения точности. Кроме того, предполагается дальнейшее расширение функционала программного модуля для обеспечения возможности обработки данных с других космических аппаратов.

\section{References}

[1] Kataev M.Yu., Bekerov A.A., Methodology of the water objects detection from multi-spectrum satellite measurements, Proceedings of TUSUR journal, 2017, part 20, №4. DOI: 10.21293/1818-0442-2017-20-4-105108. (In Russian). 
[2] Moradi M., Sahebi M., Shokri M., Modified optimization water index (MOWI) for Landsat-8 OLI/TIRS, Int. Arch. Photogramm. Remote Sens. Spatial Inf. Sci., 2017, XLII-4/W4, pp. 185-190. DOI: 10.5194/isprsarchives-XLII-4-W4-185-2017.

[3] Zhou Y., Dong J., Xiao X., Xiao T., Yang Z., Zhao G., Zou Z., Qin Y., Open surface water mapping algorithms: a comparison of water-related spectral indices and sensors, Water, 2017, 9, p. 256. DOI: $10.3390 /$ w 9040256

[4] Berkovich K.M., Ruleva S.N., Chalov R.S., River Ob, Popular science encyclopedia «Water of Russia», http://water-rf.ru/a1754. (In Russian).

[5] Loveland T.R., Irons J.R., Landsat 8: The plans, the reality, and the legacy. Remote Sensing of Environment, 2016, 185, 1-6. DOI: 10.1016/j.rse.2016.07.033

[6] Xu H., Modification of normalised difference water index (NDWI) to enhance open water features in remotely sensed imagery, International Journal of Remote Sensing, 2006, Vol. 27, No. 14, pp. 3025-3033. DOI: 10.1080/01431160600589179

[7] Hall D.K., Riggs G.A., Normalized-difference snow index (NDSI), Encyclopedia of Snow, Ice and Glaciers, 2010, pp. 779-780. DOI: 10.1007/978-90-481-2642-2_376

[8] Ji L., Zhang L., Wylie B.K., Rover J.R., On the terminology of the spectral vegetation index (NIR SWIR)/(NIR + SWIR), International Journal of Remote Sensing, 2011, Vol. 32, No. 21, pp. 6901-6909, DOI: 10.1080/01431161.2010.510811.

[9] Tucker C.J., Red and photographic infrared linear combinations for monitoring vegetation, Remote Sensing of Environment, 1979, 8, pp. 127-150. DOI: 10.1016/0034-4257(79)90013-0 